\title{
Serum CA 19-9 Levels in Benign and Malignant Diseases Associated with the Gastrointestinal Tract
}

\author{
Aseem Bhattarai ${ }^{1 *}$, Bharat Jha ${ }^{1}$, Santosh Timilsina ${ }^{1}$
}

BACKGROUND: The diagnostic utility of CA 19-9 has been thoroughly evaluated and revised more than once over the past years in the context of pathological conditions related to the gastrointestinal tract. Our study was conducted to evaluate the diagnostic usage of CA 19-9 levels in the serum of patients affected with various disorders of the gastrointestinal tract, and to assess the diagnostic values in terms of severity and organ system involved in the context of clinical disorders requiring surgical interventions.

METHODS: A total of 70 patients admitted to various surgical units and the critical care units were randomly selected and the sera analyzed for levels of CA 19-9. Mean values were compared using independent t-tests and ANOVA, and cut-offs were estimated using ROC Curves.

RESULTS: CA 19-9 levels in the serum were found to be elevated in $57 \%, 50 \%, 100 \%, 81 \%$ and $63 \%$ of patients with Stomach / Upper GI, Liver / Gall Bladder, Pancreas, Colorectal and Lungs/Pleural pathologies respectively, with the normal cut-off values at $35 \mathrm{U} / \mathrm{ml}$. In general, mean values of CA 19-9 in the serum were higher in patients with neoplasms $(75.63 \pm 34.3 \mathrm{U} / \mathrm{ml})$ compared to patients with inflammatory conditions $(44.5 \pm 20.5 \mathrm{U} / \mathrm{ml})$, $(p<0.001)$. Similarly, mean values of CA 19-9 were higher in patients with malignant neoplasms compared to benign lesions $(56.06 \pm 24.5 \mathrm{U} / \mathrm{ml}$, compared to $88.39 \pm 34.2 \mathrm{U} / \mathrm{ml}, \mathrm{p}=0.003)$. Patients with pancreatic pathologies had the highest mean values of CA $19-9$ in their serum $94.43 \pm 29.28 \mathrm{U} / \mathrm{ml}$ ) compared to patients with other organ system pathologies $(\mathrm{p}<0.001)$. When assessed separately, patients with malignant conditions of the pancreas had a higher mean value of serum CA 19-9 compared to patients with non-malignant and inflammatory conditions of the pancreas $121.0 \pm 16.7 \mathrm{U} / \mathrm{ml}$ compared to $74.5 \pm 17.86 \mathrm{U} / \mathrm{ml}, \mathrm{p}<0.001)$. Mean levels of CA 19-9 were also higher in patients with surgically inoperable stages of malignant cancers of the pancreas in comparison to patients who had a surgically resectable mass in the pancreas $(99.36 \pm 33.51$ compared to $65.91 \pm 30.11 \mathrm{U} / \mathrm{ml}, \mathrm{p}=0.02$ ). Finally, in order to establish a cut-off value for achieving maximal sensitivity and specificity to diagnose malignant conditions of the pancreas, the cut-offs were set at $92 \mathrm{U} / \mathrm{ml}$ achieving $100 \%$ sensitivity and $90 \%$ specificity.

CONCLUSIONS: The serum levels of CA 19-9 are practically elevated in all conditions associated with the Gastrointestinal System, and thus, at current cutoff values, this marker is at best, non-specific. Higher values, however, are typically associated with pancreatic conditions, both benign and malignant. Thus, with a higher cut-off value, CA 19-9 serves better as a specific marker for the diagnosis of clinical conditions of the pancreas, at preferably above 90 $\mathrm{U} / \mathrm{ml}$.

(C) 2015 Nepalese Association for Clinical Chemistry

\section{Introduction}

CA 19-9 (Carbohydrate Antigen 19-9) is regularly used as a diagnostic marker in the evaluation and monitoring of cancers associated with the pancreas over the past several years in the context of Nepal. The reported values of CA 19-9 in the serum are quite often elevated above the expected cutoff values. The purpose of our study was to observe the serum levels of CA 19-9 in patients suffering from different clinical conditions (both benign, like infections, and malignant) related to the gastrointestinal and hepatobiliary system.

It is well known now that CA19-9 is an antibody that binds to the tumor surface marker SialylLewis-A produced by cancer cells of gastrointestinal, and more specifically, pancreatic origin [1] Regardless, owing to the reported high degree of false negative and false positive cases in the context of CA 19-9 as a diagnostic marker for cancers, the American Society of Clinical Oncology Guidelines implicitly discourage the use of CA19-9 as a screening test furthermore, particularly pancreatic cancer. Current practice dictates the main use of CA19-9 in establishing confirmation on first, whether a pancreatic tumor is secreting it at all. If so, the levels should visibly fall when the tumor is treated, and they may increase in levels on recurrence of the lesion [2]. CA 19-9, thus, serves better as a prognostic indicator in patients with pancreatic tumors.

Several studies have been conducted over the past years trying to establish the diagnostic and prognostic value of the marker (CA 19-9) both

'Department of Biochemistry, Maharjgunj Medical Campus, IOM, TUTH, Nepal. 


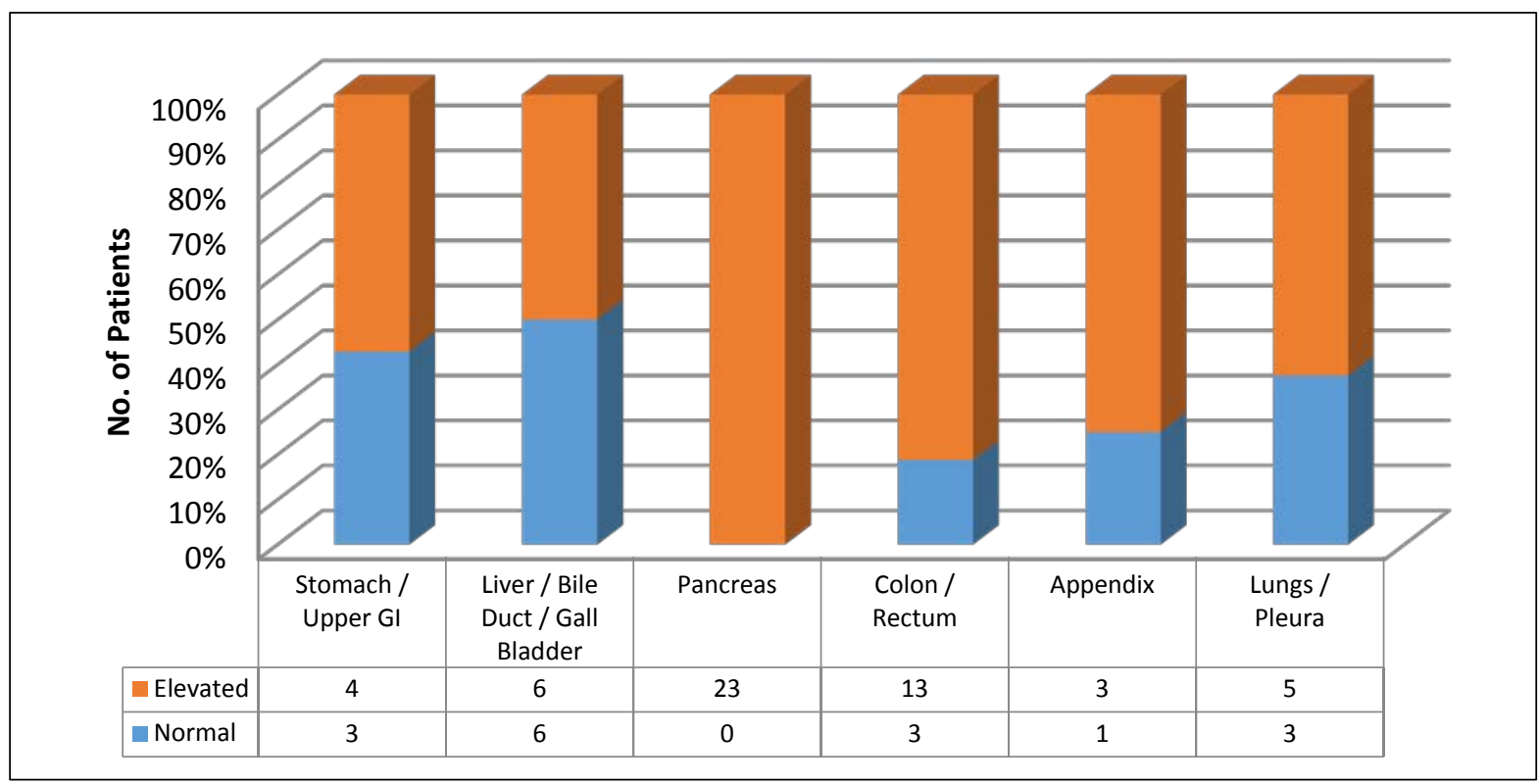

Figure. 1. Categorized Serum CA 19-9 Levels and organ system involved

Table 1. Comparison of Mean values of CA 19-9 across different patient groups.

\begin{tabular}{lllll}
\hline & Patient Group & (n) & Mean \pm S.D. & p-value \\
\hline \multirow{2}{*}{ Pathology Involved } & Infection & 32 & $44.5 \pm 20.5$ & \multirow{2}{*}{0.001} \\
& Neoplasm & 38 & $75.63 \pm 34.3$ & \multirow{2}{*}{ Neoplasm Type } \\
& Benign & 15 & $56.06 \pm 24.5$ & 0.003 \\
\hline \multirow{2}{*}{ Ascites } & Malignant & 23 & $88.39 \pm 34.2$ & \multirow{2}{*}{ Present } \\
\multirow{2}{*}{ Tumor State } & Absent & 24 & $85.04 \pm 34.65$ & \multirow{2}{*}{0.001} \\
& Operable & 46 & $49.06 \pm 23.81$ & \\
\hline
\end{tabular}

singly and in combination with other known tumor markers. Some of these studies have been focused on establishing the predictive values and sensitivity of detection of different cancers of the gastrointestinal tract [3]. Other studies have been conducted to evaluate the use of this marker in the context of inflammatory processes of the hepatobiliary system, like cholangitis [4] and pancreatitis [5], for example.

Based upon the studies conducted over the years, the establishment of a diagnosis based upon CA 19-9 values seems to be rather difficult, because elevated values are seen in a diverse range of disorders, including but not limited to colorectal cancers and inflammatory diseases of the colon [6], [7], gastrointestinal cancers [8], upper gastrointestinal diseases [9], pleural effusions [10] and ascites [11].

As a rule, serum levels of CA 19-9 are ordered to confirm a clinical suspicion of pancreatic cancers in our context. We intended to assess whether the values of CA 19-9 are significantly elevated in the context of other disorders as well. If the values were to be elevated as suspected and shown by contemporary researchers, we also planned to assess whether a higher cutoff value for the marker would increase specificity of detection for malignant pancreatic conditions compared to other disorders.

The use of CA 19-9 as an adjunct in combination with other markers was suggested by CarpelanHolmström $M$ et.al [8] in the late nineties, where CEA, CA 19-9, CA 242 and CA 72-4 levels were measured in patients with benign and malignant gastrointestinal diseases and a logistic regression model was deployed to assess the diagnostic value (concomitant) of these markers. The conclusion was that the algorithm based on the combination of CEA, CA 19-9 and CA 72-4 improved the diagnostic accuracy in detection of gastrointestinal tract malignancies compared to using the markers alone [8]. The use of CA 19-9 in combination with other markers is not relevant in our scenario 
because all tumor markers are not practically established as easy access screening tools yet, both for economic reasons and lack of specialized laboratories nationwide.

Table 2. Comparison of mean values of CA 19-9 across different organ systems.

\begin{tabular}{lccc}
\hline $\begin{array}{l}\text { Organ System } \\
\text { Involved }\end{array}$ & Mean \pm S.D. & (n) & p-value \\
\hline $\begin{array}{l}\text { Stomach/Upper GI } \\
\text { Liver/Bile Duct/Gall }\end{array}$ & $37.71 \pm 16.2$ & 7 & \\
Bladder & $94.43 \pm 29.28$ & 23 & \\
Pancreas & $54.8 \pm 22.38$ & 16 & $<0.001$ \\
Colon/Rectum & $50.0 \pm 27.8$ & 4 & \\
Appendix & $45.5 \pm 14.29$ & 8 & \\
Lungs/Pleura & & & \\
\hline
\end{tabular}

The notion that using a higher cutoff value for CA 19-9 could improve the specificity of detection of pancreatic cancers has also been suggested by Steinberg W, [3] where he concluded that with higher cutoff values, specificities of $100 \%$ can be achieved in the context of pancreatic cancer. Very high values, i.e. above $1000 \mathrm{U} / \mathrm{ml}$ were associated with high grade and non resectable tumors of the pancreas [3] This is similar to the findings expressed by Ritts et al. [12] in their evaluation of CA 19-9 in the context of pancreatic conditions, where higher values (above $200 \mathrm{U} / \mathrm{ml}$ ) are associated with malignant diseases and preoperative values served as prognostic indicators of pancreatic cancers. With the establishment of a higher cutoff value to the serum levels of CA 19-9, the diagnostic utility would be greatly increased, providing an insight to the attending surgeon about the condition with the use of a screening marker, thus increasing efficiency. Other researchers have evaluated the use of CA 19-9 in the differential diagnosis of chronic pancreatitis and pancreatic carcinomas in the past. Del Maschio et al. [5] concluded that the diagnostic value of CA 19-9 was best for malignant conditions of the pancreas, according to their study, although the best means was clearly FNAB (CT guided), with the best positive predictive value in diagnosis [5].

Some researchers have been focused on establishing the prognostic value of CA 19-9 in addition to the diagnostic value, like in the research conducted by Patel et al. [4], assessed the diagnostic utility of CA 19-9 in relation to cholangiocarcinoma and found that the sensitivity of a CA 19-9 value $>100 \mathrm{U} / \mathrm{ml}$ in diagnosing cholangiocarcinoma was $53 \%$. Similar to what Steinberg states, Patel also discovered that patients with unresectable cholangiocarcinoma had significantly greater mean serum values of CA 1994, suggesting that CA 19-9 values are also an indicator of prognosis in patients with

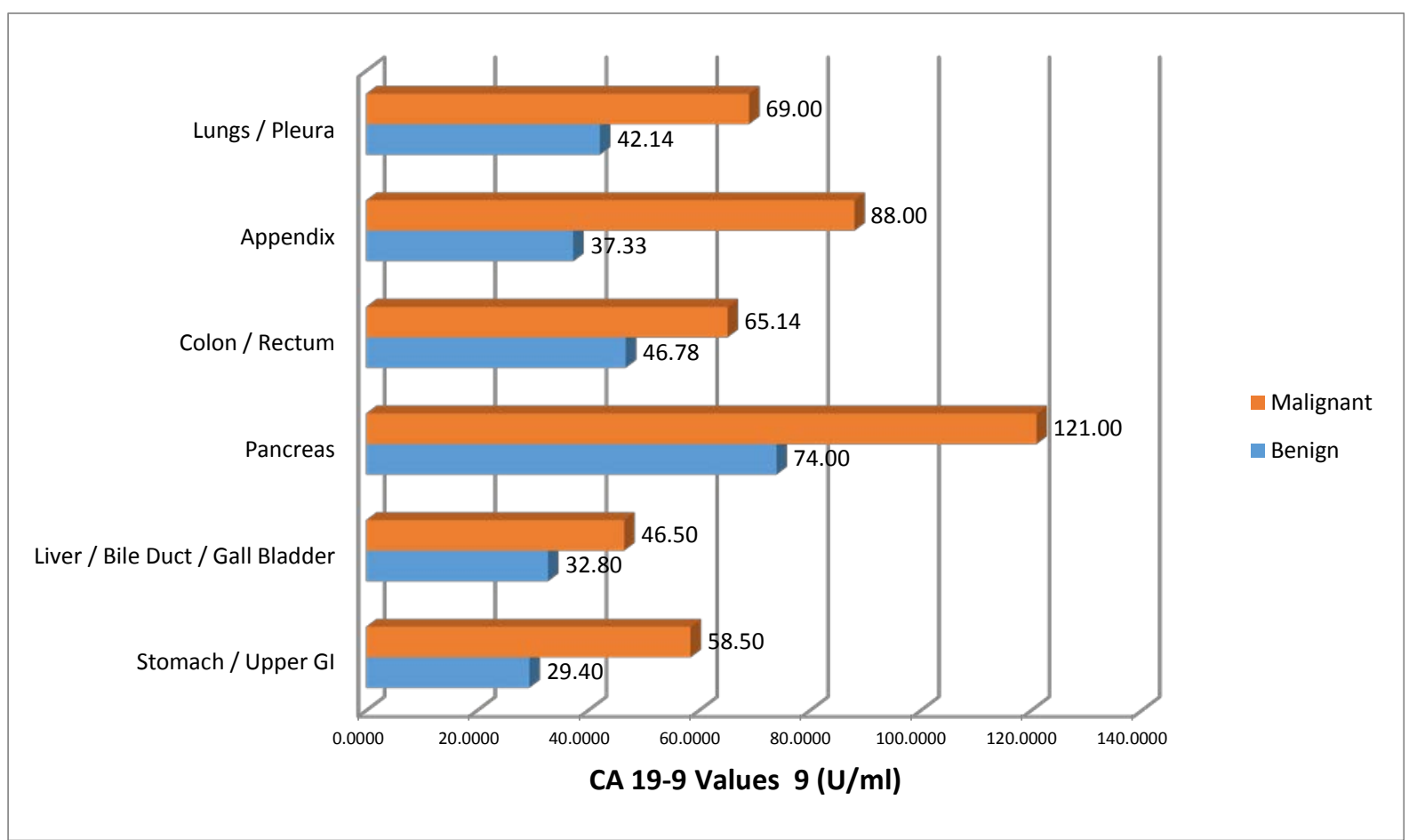

Fig. 2: Relative values of CA 19-9 and organ system involved. 
gastrointestinal cancers and this reinforces the fact that serum CA 19-9 levels can be used not just as a diagnostic tool but also as a prognostic indicator in certain tumors of the Gastrointestinal Tract.

In a similar study conducted by Okzan et al. [13], the diagnostic values of CA 242 was compared against that of CA 19-9 in the diagnosis of pancreatic cancer, and although the diagnostic values were good in case of both the markers, CA 242 had better specificity compared to CA 19-9 ( $85 \%$ vs. $67 \%$ respectively) with similar sensitivity values at regular cutoff intervals [13].

CA 19-9 levels have also been used to screen cancers of the biliary tract with or without cholangitis. Levy et al. [14] state in their research paper that a cutoff of $63.2 \mathrm{U} / \mathrm{ml}$ for change in CA $19-9$ provided a sensitivity of $90 \%$ and a specificity of $98 \%$ with a positive predictive value of $42 \%$ in the diagnosis of cholangiocarcinoma in patients with sclerosing cholangitis [14].

Also, in people with pancreatic masses that are visible on imaging, serum values of CA19-9 can be used in differentiating pancreatic cancer from other diseases of the gland $[1,15]$.

CA 19-9 has also been assessed for use in combination with other markers like CEA, CA 15-3, NSE (Neuron Specific Enolase) and CYFRA (Cytokeratin Fragments) in the diagnosis of pleural effusions, as shown by Alatas F et al. [10] in his research. This marker has also been used in the diagnosis of colorectal cancers as stated by Reiter et al [6], in his research.

Our study, thus, was conducted to assess the diagnostic utility of serum CA 19-9 values in the diagnosis of various gastrointestinal conditions.

\section{Methods}

A cross-sectional study was conducted in 70 patients admitted with the diagnosis of gastrointestinal cancers (histologically or radiologically established) and other clinical conditions (infections and benign lesions) related to the gastrointestinal tract, and their blood samples analyzed for levels of CA 19-9. All samples were run on an internal standardization based automated enhanced chemiluminescent immunoassay (Vitros $®$ ECi) that uses HRPlabeled antibodies as signal reagents. Patients were divided into groups based upon the organ system involved and selected by random sampling from the surgical and critical care units.

We obtained the Coefficient of Variation by comparing the test results for the first ten samples when run stat, in twenty four hours and at the end of the week. A CV of less than $10 \%$ was considered acceptable and the mean $\mathrm{CV}$ was $6.76 \%$. For the rest of the study, serum samples were stored at $4^{\circ} \mathrm{C}$ and batches run at the end of the week. The Data was analyzed using IBM SPSS 20.0, and mean values were compared across different groups of patients using Analysis of Variance (ANOVA). Cutoffs were estimated using receiver operator characteristics curve (ROC Curve) analysis.

Table 3. Pancreatic Pathology and Mean CA 19-9 Levels.

\begin{tabular}{lccc}
\hline $\begin{array}{l}\text { Pancreatic } \\
\text { Pathology }\end{array}$ & (n) & Mean \pm S.D. & p-value \\
\hline Benign & 12 & $74.5 \pm 17.86$ & \\
Malignant & 10 & $121.0 \pm 16.7$ & $<0.001$ \\
\hline
\end{tabular}

Table 4. Surgical State of Pancreatic neoplasm and Mean CA 19-9 Levels.

\begin{tabular}{lccc}
\hline $\begin{array}{l}\text { Surgical Stage of } \\
\text { Tumor }\end{array}$ & $(\mathrm{n})$ & Mean \pm S.D. & p-value \\
\hline Operable & 10 & $93.1 \pm 27.0$ & \multirow{2}{*}{0.02} \\
Inoperable & 6 & $124.83 \pm 16.1$ & \\
\hline
\end{tabular}

\section{Results}

Our study included similar number of patients in the infection group (45\%) and the neoplasm group (55\%). We had, however, more male subjects $(61 \%, \mathrm{n}=43)$ in our study as compared to females $(39 \%, n=27)$. We had the highest number of patients with pancreatic pathologies $(32 \%)$ as compared to other organ systems in our study.

Most patients included in the study had elevated levels of CA 19-9, with the exception of a few patients with infections who had clinically acceptable (normal) levels of CA 19-9 in their serum (i.e. $<35 \mathrm{U} / \mathrm{ml}$ ).

As shown in figure 2, CA 19-9 levels were categorized into normal and elevated with a cutoff value of $35 \mathrm{U} / \mathrm{ml}$, and a cross tabulation showed that all patients with pancreatic lesions had elevated values of CA 19-9 in their sera. Exactly $50 \% \quad(n=6)$ of the patients with pathologies involving the hepatobiliary system, otherwise had elevated levels of the same. To summarize, the CA 19-9 levels in the serum were found to be elevated in $57 \%, 50 \%, 100 \%, 81 \%$ and $63 \%$ of patients with Stomach / Upper GI, Liver / Gall Bladder, Pancreas, Colorectal and Lungs/Pleural pathologies respectively. Thus, a larger proportion 
of patients with clinical conditions involving the GI tract and pleural effusions had elevated levels of CA 19-9 in their serum in our study.

As shown in table 2, patients were grouped according to the pathology involved, neoplasm type (where relevant), whether or not there was ascites, and according to the surgical state of the neoplasm (where relevant), and mean values of CA 19-9 between patients within groups. A subsequent t-test showed that the values of CA $19-9$, in general, was significantly higher (75.63 \pm $34.3 \mathrm{U} / \mathrm{ml}$ ) in patients with neoplasm as compared to those with infection $(44.5 \pm 20.5$ $\mathrm{U} / \mathrm{ml}),(\mathrm{p}<0.001)$. Similarly, the mean values of CA 19-9 were significantly elevated in patients with malignant neoplasms compared to benign lesions ( $\mathrm{p}=0.003)$, higher in patients with ascites $(\mathrm{p}<0.001)$ and significantly elevated in patients with inoperable lesions $(99.36 \pm 33.51 \mathrm{U} / \mathrm{ml})$ compared to patients with surgically resectable tumors $(65.91 \pm 30.11 \mathrm{U} / \mathrm{ml}), \quad(\mathrm{p}=0.02)$ respectively.

When compared across groups of patients (according to organ system involved), Serum levels of CA 19-9 were found to be significantly elevated in patients with pancreatic lesions, as compared to patients in other groups $(\mathrm{p}<0.001)$. This did reveal, however, that other organ system pathologies could also cause an increase in CA 199 levels, albeit to a very small degree compared to pancreatic pathologies (table 2). Malignant conditions related to all organ systems had average values of CA 19-9 that were higher than that for benign lesions of the same organ system as shown in figure 2 .

When we looked at pancreatic pathologies as a single group, divided patients into two groups based on their disease type (benign and malignant) and compared the mean values of CA 19-9 between patients in these groups, it was seen that patients with malignant lesions of the pancreas (most commonly adenocarcinomas) had significantly higher levels of CA 19-9 (121.0 \pm $16.7 \mathrm{U} / \mathrm{ml}$ vs. $74.5 \pm 17.86 \mathrm{U} / \mathrm{ml}),(\mathrm{p}<0.001)$, respectively (table 3 ).

Also, patients with surgically resectable tumors of the pancreas had a slightly lower, albeit significant mean value of Serum CA 19-9 levels ( $p=0.02$, table 4).

We also grouped the remaining patients (patients with non-pancreatic pathologies) according to the neoplasm type (benign and malignant) and compared the mean values of CA 19-9 across groups for each organ system (Table 5). It was seen, however, that although the values looked different in patients with malignant lesions as compared to that of non-malignant lesions, the difference was not statistically evident or significant at the $\mathrm{p}=0.001$ level.

We also conducted a follow up receiver operator characteristics (ROC) curve analysis to evaluate the cutoff values for CA 19-9 in order to be able to screen malignant conditions of the pancreas. With a cutoff value of $92 \mathrm{U} / \mathrm{ml}$, our study showed that one can achieve 100\% sensitivity and 90\% specificity for detection of malignant pancreatic neoplasms (AUC 0.92, p< 0.001). At lower cutoff values, like $51 \mathrm{U} / \mathrm{ml}$, for instance, the sensitivity was still $100 \%$, as expected, but the specificity decreased to less than $20 \%$. The number of false positives due to several other conditions in the gastrointestinal tract could clearly not be overlooked at this point, and this supported the values of CA 19-9 we found in the serum samples of other clinical conditions in our study.

\section{Discussion}

In the context of upper gastrointestinal tract associated cancers, a study was conducted by Jalanko et al. [9], in which the team evaluated the levels of CA 19-9 in patients with benign and malignant gastrointestinal cancers. They found

Table 5. Pathological Subtypes and Mean CA 19-9 Levels

\begin{tabular}{|c|c|c|c|}
\hline \multirow{2}{*}{ Organ System } & \multicolumn{2}{|c|}{ Pathology Subtype } & \multirow{2}{*}{ p-value } \\
\hline & Benign & Malignant & \\
\hline \multirow{2}{*}{ Stomach / Upper GI } & $29.4 \pm 9.31$ & $58.5 \pm 4.94$ & \multirow{2}{*}{ NS } \\
\hline & $\mathrm{n}=5$ & $\mathrm{n}=2$ & \\
\hline \multirow{2}{*}{ Liver / Bile Duct / Gall Bladder } & $32.8 \pm 10.17$ & $46.5 \pm 2.12$ & \multirow{2}{*}{ NS } \\
\hline & $\mathrm{n}=10$ & $\mathrm{n}=2$ & \\
\hline \multirow{2}{*}{ Colon / Rectum } & $46.7 \pm 19.8$ & $65.14 \pm 22.5$ & \multirow{2}{*}{ NS } \\
\hline & $\mathrm{n}=9$ & $\mathrm{n}=7$ & \\
\hline \multirow{2}{*}{ Appendix } & $37.33 \pm 14.15$ & $88.0 \pm 0.0$ & \multirow{2}{*}{ NS } \\
\hline & $\mathrm{n}=3$ & $\mathrm{n}=1$ & \\
\hline \multirow{2}{*}{ Lungs / Pleura } & $42.1 \pm 11.53$ & $69.0 \pm 0.0$ & \multirow{2}{*}{ NS } \\
\hline & $\mathrm{n}=7$ & $\mathrm{n}=1$ & \\
\hline
\end{tabular}


that the CA 19-9 concentrations were above the upper limit of the normal range $(0-37 \mathrm{U} / \mathrm{ml})$ in $76 \%$ of patients with pancreatic carcinoma, $73 \%$ of patients with cholangiocarcinoma, $42 \%$ of patients with gastric carcinoma, and $22 \%$ of patients with hepatoma [9]. Our results showed a similar pattern with $100 \%$ of patients with pancreatic condtions having elevated CA 19-9 levels, with proportion of patients with elevated CA $19-9$ at $55 \%$ for gastric conditions, $50 \%$ for hepatobiliary conditions, $70 \%$ for colorectal conditions and close to $70 \%$ for pleural and appendicular conditions. This established the fact that at this cutoff value (i.e. $35 \mathrm{U} / \mathrm{ml}$ ) the diagnostic value of CA 19-9 becomes highly compromised, with elevations seen in almost all conditions involving the GI tract, and the specificity is practically very low, enforcing the fact that a higher cutoff needs to be established for improving diagnostic applications.

Similar to the findings shown by CarpelanHolmström M et. al [8] in their study, where they found increased levels of CA 19-9 in different proportions of patients with pancreatic, biliary and hepatic diseases, our study also showed that at the commonly accepted cutoff values, the diagnostic significance decreases, except in the case of pancreatic cancers (adenocarcinomas), where the test can be pretty much specific at higher cutoff values ( $95 \mathrm{U} / \mathrm{ml}$ or higher).

Just like Steinberg [3] mentioned in his study, higher mean values of CA 19-9 were found to be associated with higher surgical stages of the tumors of the pancreas, as shown by our study $(93.1 \pm 27.0 \mathrm{U} / \mathrm{ml}$ in operable cases of pancreatic cancer compared to $124.83 \pm 16.1 \mathrm{U} / \mathrm{ml}$ in surgically inoperable cases, $\mathrm{p}=0.02$ ). Similarly, the mean serum values of CA 19-9 were significantly higher in patients with malignant pancreatic lesions compared to benign lesions (pancreatitis, pseudocysts etc.) at $121.0 \pm 16.7 \mathrm{U} / \mathrm{ml}$ in malignant conditions as compared to $74.5 \pm$ $17.86 \mathrm{U} / \mathrm{ml}, \mathrm{p}<0.001$. This is similar to the results obtained by other researchers $[9,12,13]$.

Our study also showed that the mean values of CA 19-9 were significantly higher $(p<0.05)$ in the group of patients with Ascites, regardless of the cause, compared to the group of patients without ascites at the time of evaluation. With reference to the results obtained by Sari et al. [11], the diagnostic utility of Serum CA 19-9 in the etiology of ascites was not evaluated in our study, despite the fact that serum values were found to be elevated in patients with Ascites compared to those without.
In the study done by Alatas $\mathrm{F}$ et al., there was a significant rise of all tumor markers (including CA 19-9) in the sera of patients with malignant pleural effusions, as well as in the specific pleural fluid samples. CA 19-9, with a cutoff of $5 \mathrm{U} / \mathrm{ml}$ in pleural fluid samples gathered a sensitivity of $36 \%$ in detecting pleural fluid malignancies, while the specificity was at $83 \%$ [10]. This is in par with the findings of our study where the mean values of serum CA 19-9 were elevated at 69.0 $\mathrm{U} / \mathrm{ml}$ in the serum of patients with malignant effusions compared to $42.1 \pm 11.5 \mathrm{U} / \mathrm{ml}$ in patients with pleural effusions due to benign conditions. These values, however, do not have a statistical significance in terms of diagnostic values to establish a causative diagnosis (malignant or otherwise) of pleural effusions, according to our study.

Reiter et al. [6], in their research, evaluated the prognostic value of CEA and CA 19-9 in the management of colorectal cancers. Preoperative CA 19-9 values were found to offer valuable clinical insight into the outcome of the colorectal cancer with cutoffs above $60 \mathrm{U} / \mathrm{ml}$ being associated with a decreased survival time. Mean values of CA 19-9 in patients with colorectal cancers were found to be $65.14 \pm 22.5 \mathrm{U} / \mathrm{ml}$ in our study, (the wide SD attributed to the different stages of colorectal cancers included in the research), which was significantly higher than the mean $46.7 \pm 19.8 \mathrm{U} / \mathrm{ml}$ seen in benign lesions of the colon/rectum areas. In addition, Kuusela [7], states in his research, that CA 19-9 is a better suited marker in terms of specificity compared to CEA in the diagnosis of Colorectal Cancers [7]. Our study was more focused on the diagnostic value of CA 19-9, and although the difference seen in values between the two groups of patients (benign and malignant) is obvious, owing to the small sample size of patients with Colorectal Carcinomas, the results were not statistically significant $(\mathrm{p}>0.05)$.

\section{Conclusion}

The serum levels of CA 19-9 are practically elevated in all conditions associated with the Gastrointestinal System, and thus, at current cutoff values, this marker is at best, non-specific. Higher values, however, are typically associated with pancreatic conditions, both benign and malignant and hence, with a higher cutoff value, preferably above $90 \mathrm{U} / \mathrm{ml}$, can potentially serve as a specific marker for the diagnosis of pancreatic disorders. 
Conflict of Interest: None declared

\section{REFERENCES}

1. Perkins GL, Slater ED, Sanders GK, Prichard JG. Serum tumor markers. American family physician. 2003;68(6):1075-82.

2. Locker GY, Hamilton S, Harris J, Jessup JM, Kemeny N, Macdonald JS, et al. ASCO 2006 update of recommendations for the use of tumor markers in gastrointestinal cancer. Journal of clinical oncology : official journal of the American Society of Clinical Oncology. 2006;24(33):5313-27

3. Steinberg W. The clinical utility of the CA 19-9 tumor-associated antigen. The American journal of gastroenterology. 1990;85(4):350-5.

4. Patel AH, Harnois DM, Klee GG, LaRusso NF, Gores GJ. The utility of CA 19-9 in the diagnoses of cholangiocarcinoma in patients without primary sclerosing cholangitis. The American journal of gastroenterology. 2000;95(1):204-7.

5. DelMaschio A, Vanzulli A, Sironi S, Castrucci M, Mellone R, Staudacher $\mathrm{C}$, et al. Pancreatic cancer versus chronic pancreatitis: diagnosis with CA 19-9 assessment, US, CT, and CT-guided fine-needle biopsy. Radiology. 1991;178(1):95-9.

6. Reiter W, Stieber P, Reuter C, Nagel $\mathrm{D}$, Lau-Werner U, Lamerz R. Multivariate analysis of the prognostic value of CEA and CA 19-
9 serum levels in colorectal cancer. Anticancer research. 2000;20(6D):5195-8.

7. Kuusela P, Jalanko H, Roberts P, Sipponen P, Mecklin JP, Pitkanen $\mathrm{R}$, et al. Comparison of CA 19-9 and carcinoembryonic antigen (CEA) levels in the serum of patients with colorectal diseases. British journal of cancer. 1984;49(2):135-9.

8. Carpelan-Holmstrom M, Louhimo J, Stenman UH, Alfthan $\mathrm{H}$, Haglund C. CEA, CA 19-9 and CA 72-4 improve the diagnostic accuracy in gastrointestinal cancers. Anticancer research. 2002;22(4):2311-6.

9. Jalanko H, Kuusela P, Roberts P, Sipponen P, Haglund CA, Makela $O$. Comparison of a new tumour marker, CA 19-9, with alphafetoprotein and carcinoembryonic antigen in patients with upper gastrointestinal diseases. Journal of clinical pathology. 1984;37(2):21822. Epub 1984/02/01.

10. Alatas F, Alatas O, Metintas M, Colak O, Harmanci E, Demir S. Diagnostic value of CEA, CA 15-3, CA 19-9, CYFRA 21-1, NSE and TSA assay in pleural effusions. Lung Cancer. 2001;31(1):9-16.

11. Sari R, Yildirim B, Sevinc A, Bahceci F, Hilmioglu F. The importance of serum and ascites fluid alpha- fetoprotein, carcinoembryonic antigen, CA 19-9, and CA 15-3 levels in differential diagnosis of ascites etiology. Hepatogastroenterology. 2001;48(42):161621.

12. Ritts RE, Pitt HA. CA 19-9 in pancreatic cancer. Surgical oncology clinics of North America. 1998;7(1):93-101.

13. Ozkan H, Kaya M, Cengiz A. Comparison of tumor marker CA 242 with CA 19-9 and carcinoembryonic antigen (CEA) in pancreatic cancer. Hepatogastroenterology. 2003;50(53):166974.

14. Levy C, Lymp J, Angulo P, Gores GJ, Larusso N, Lindor KD. The value of serum CA 19-9 in predicting cholangiocarcinomas in patients with primary sclerosing cholangitis. Digestive diseases and sciences. 2005;50(9):1734-40.

15. Goonetilleke KS, Siriwardena AK. Systematic review of carbohydrate antigen (CA 19-9) as a biochemical marker in the diagnosis of pancreatic cancer. European journal of surgical oncology: the journal of the European Society of Surgical Oncology and the British Association of Surgical Oncology. 2007;33(3):266-70. 